\title{
Leishmaniose tegumentar americana: flebotomíneos em área de transmissão no Município de Uberlândia, MG
}

\author{
American cutaneous leishmaniasis: phlebotomine transmission area in the \\ Municipality of Uberlândia, MG
}

\author{
Jureth Couto Lemos ${ }^{1}$ e Samuel do Carmo Lima ${ }^{2}$
}

\begin{abstract}
RESUM0
De maio de 2000 a janeiro de 2001, foram realizadas 4 capturas de flebotomíneos em área de transmissão da leishmaniose tegumentar americana na bacia do rio Araguari, no Município de Uberlândia, MG, com duração de 15 horas consecutivas cada, para observar o comportamento dos insetos. A primeira captura foi em maio de 2000 (frio e úmido), a segunda em junho de 2000 ( frio e seco), a terceira em outubro de 2000 (quente e seco) e a quarta em janeiro de 2001 (quente e chuvoso). Utilizaram-se para capturar os flebótomos armadilhas Center on Disease Control e Shannon. Foram capturados e identificados 6551 flebótomos, sendo 1990 machos e 4561 fêmeas distribuídos em 2 gêneros (Lutzomyia e Brumptomyia) e 8 espécies. A Lutzomyia intermedia predominou com o maior número de espécimens (6531), representando 99,7\% dos flebótomos capturados. Nas quatro capturas observou-se a preferência de Lutzomyia intermedia pelo mês que precede o período chuvoso (outubro), com temperaturas e umidade relativa do ar altas.
\end{abstract}

Palavras-chaves: Leishmaniose tegumentar americana. Fauna flebotomínica. Phlebotominae.

\begin{abstract}
From May 2000 to January 2001 four, 15 hour-long collections of phlebotomine were done to the insect monitoring. The collection was done in an area of infection of American cutaneous leishmaniasis in the basin of the Araguari River in the municipality of Uberlândia (MG).The first collection was made in May (a cold, damp month), the second in June 2000 (a cold, dry month) the third in October 2000 (a hot, dry month) and the fourth in January 2001 (a hot, rainy month).CDC and Shannon light traps were used 6551 phlebotomne were captured and identified, 1990 male and 4562 female, comprised of two lines (Lutzomyia and Brumptomyia) and 8 species. Lutzomyia intermedia predominated with the largest number of specimens (6531), which accounted for 99,7\% of the collected insects. In the four collections, it was observed that Lutzomyia intermedia manifested a preference for the month preceding the rainy season, with its high temperatures and humidity.
\end{abstract}

Key-words: American cutaneous leishmaniasis. Phlebotomine sandflies fauna. Phlebotominae.

A leishmaniose tegumentar americana ( LTA) constitui importante problema de saúde pública em vários Estados brasileiros. Encontra-se em franca expansão e, longe de ter um controle eficiente da doença. Na década de 50, houve uma diminuição geral da ocorrência da parasitose, porém, o número de casos vem aumentando progressivamente nosúltimos 20 anos, observando-se surtos em todas as regiões do país. Em Minas Gerais, no período de 1997 a 1999, houve um aumento de casos de $44,3 \%$, deixando de ser considerado de Baixo coeficiente de detecção/100.000hab <3,00 em 1985 para Alto 11,00<71,00 em 19997.

A expansão agropecuária nas áreas de Cerrados do Brasil Central vem sendo acompanhada por aumento de casos humanos de leishmaniose tegumentar americana ${ }^{8}$. Nestas áreas, a LTA que antes era restrita a áreas rurais, agora se faz presente nos centros urbanos, atingindo populações menos favorecidas ${ }^{12}$.

1. Escola Técnica de Saúde da Universidade Federal de Uberlândia, Uberlândia, MG. Uberlândia, MG. 2. Instituto de Geografia da Universidade Federal de Uberlândia, Uberlândia, MG.

Endereço para correspondência: Dra. Jureth Couto Lemos. Universidade Federal de Uberlândia. Rua Antônio Salviano de Rezende 1153, Santa Mônica, 38408-224 Uberlândia, MG

Tel: 34 3212-3979

e-mail: jclemos@ufu.br

Recebido em 15/1/2003

Aceito em 16/11/2004 
0 aumento da população de vetores da LTA tem como fatores favoráveis a umidade e as temperaturas elevadas, tendo implicação direta com risco de infecção numa determinada região. 0 clima quente e úmido, a declividade (boqueirões), fontes de alimento e outros parâmetros ecológicos determinam a distribuição e a possível ocorrência do ciclo da doença.

Com a ocorrência da LTA endêmica no país, a notificação de casos autóctones e a falta de estudos sobre flebotomíneos em Uberlândia, houve motivação para a realização deste trabalho que visa demonstrar a fauna destes vetores e seu comportamento em área de transmissão da doença neste Município.

\section{MATERIAL E MÉTOD0S}

Características gerais do Município de Uberlândia. 0 Município de Uberlândia está localizado na porção sudoeste do Estado de Minas Gerais, no Triângulo Mineiro, entre as coordenadas geográficas de $18^{\circ} 30^{\prime}-19^{\circ} 30^{\prime}$ de latitude sul e $47^{\circ} 50^{\prime}$ - 4850 longitude oeste de Greenwich, no domínio dos Planaltos e Chapadas da Bacia Sedimentar do Paraná, a uma altitude média de $900 \mathrm{~m}$, ocupando uma área de $4.040 \mathrm{~km}^{2}$ ( Figura 1). Sua vegetação natural é 0 cerrado do tipo savana arbórea com mata de galeria ${ }^{2111415}$.

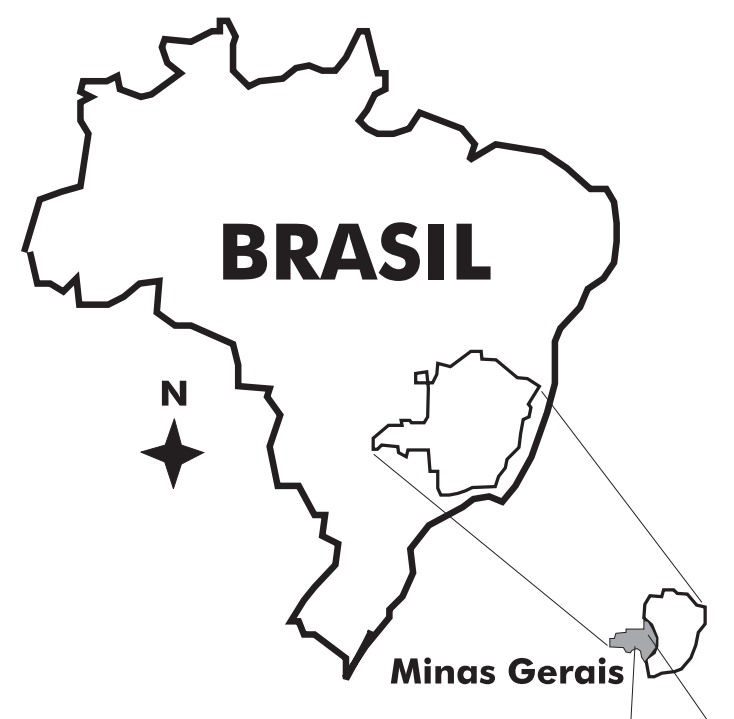

Sem escala

Fonte: Lemos et al, $2004^{10}$

Uberlândia

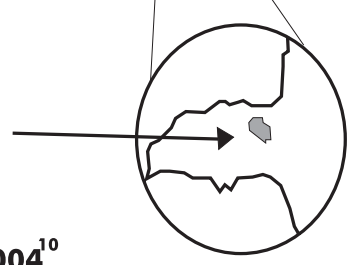

Figura 1 - Localização da área de estudo.

Em 2002, sua população era de 501.214 habitantes, com uma densidade demográfica de $121,8 \mathrm{hab} / \mathrm{km}^{2}$, sendo que 488.982 residiam na zona urbana e 12.232 na zona rura ${ }^{16}$.

0 estudo foi iniciado pelo levantamento das fichas dos pacientes diagnosticados com leishmaniose tegumentar americana ( casos autóctones) , junto a Vigilância Epidemiológica da Secretaria Municipal de Saúde de Uberlândia. A partir dessas fichas, foram identificados 25 casos de LTA em 11 locais. Nestes locais, foram realizadas capturas de 3 horas cada de outubro de 1999 a janeiro de 2001.

Dentre os 11 locais onde ocorreram casos da ITA, selecionouse aleatoriamente uma das áreas para realizar capturas de flebotomíneos com 15 horas consecutivas para observar 0 comportamento dos insetos sem levar em consideração o número de casos humanos de LTA. Este local fica a 48Km da cidade de Uberlândia, à margem esquerda do rio Araguari. É uma fazenda tomada de pastagem com várias casas à margem deste rio para atividades de lazer, como a pesca.

Neste local, foram realizadas 4 capturas de 15 horas consecutivas iniciando às 16 horas e encerrando às 7 horas do dia seguinte. As 4 capturas de 15 horas aconteceram em 4 diferentes épocas do ano. A primeira captura foi realizada nos dias 8 e 9 de maio 2000 (frio úmido), a segunda em 29 e 30 de junho de 2000 (frio seco), a terceira em 5 e 6 de outubro de 2000 (quente seco) e a quarta, em 17 e 18 de janeiro de 2001 (quente chuvoso). Foram 78 horas de captura. Os dados de temperatura e de umidade relativa do ar foram tomados no início (18 horas) e no término das capturas ( 7 horas).

Para capturar os flebótomos foram utilizadas armadilhas do tipo Shannon ${ }^{17}$ com fonte de luz de lampião a gás de 100 velas, armadilha luminosa do tipo $\mathrm{CDC}^{18}$ alimentada com baterias de 12 volts cada e 0 tubo de sucção capturador de Castro ${ }^{5}$.

A armadilha de Shannon foi instalada próxima a uma casa que é utilizada pelo proprietário nos finais de semana para repouso e lazer e as 3 armadilhas de CDC foram instaladas a aproximadamente $50 \mathrm{~m}$ de distância uma da outra como também da armadilha de Shannon.

A opção em utilizar os dois tipos de armadilhas foi para verificar a quantidade e a preferência entre 0 sexo dos flebótomos em cada armadilha.

Participaram destas capturas 5 pessoas que se revesavam durante a noite, mantendo-se sempre 3 pessoas capturando, a cada hora.

\section{RESULTAD0S}

Foram identificados 6.551 flebótomos de 2 gêneros (Brumptomyia e Lutzomyia) e 8 espécies. Aespécie com maior número de exemplares foi a Lutzomyia intermedia, com 6531, seguida de 9 espécimens de B. sp (espécie não identificada), L lutziana 4, L lenti e L sp com 2 exemplares cada, L sordellii, L whitmani e Lsallesi com um flebótomo cada. Destes, 1990 foram machos e 4.561 fêmeas (Tabelas 1 e 2).

A captura realizada das 16 horas do dia 08/05/2000 às 7 horas do dia 09/05/2000 apresentou temperatura local que foi de $21^{\circ} \mathrm{C}$ às 16 horas e $14^{\circ} \mathrm{C}$ às 7 horas, com umidade relativa do ar de $70 \%$ e $90 \%$ nos mesmos horários, sem precipitação atmosférica diária e mensal. Nesta captura, foram coletados flebótomos na armadilha tipo Shannon a 


\begin{tabular}{|c|c|c|c|c|}
\hline Data & Espécies & Machos & Fêmeas & Total \\
\hline 8 a 9 de maio de 2000 & L intermedia & 8 & 5 & 13 \\
\hline \multirow[t]{2}{*}{29 a 30 de junho de 2000} & L intermedia & - & 9 & 9 \\
\hline & L.intermedia & 833 & 532 & 1365 \\
\hline \multirow[t]{3}{*}{5 a 6 de outubro de 2000} & B.sp. & 3 & 2 & 5 \\
\hline & L whitmani & 1 & - & 1 \\
\hline & L sp & - & 2 & 2 \\
\hline \multirow[t]{3}{*}{17 a 18 de janeiro de 2001} & L intermedia & 96 & 194 & 290 \\
\hline & L lenti & 1 & 1 & 2 \\
\hline & L sallesi & - & 1 & 1 \\
\hline
\end{tabular}

Tabela 2 - Flebotomíneos capturados na armadilha de Shannon em monitoramento de 4 capturas com 15 horas consecutivas cada, em área de transmissão da leishmaniose tegumentar americana no Município de Uberlândia - MG, 2002.

\begin{tabular}{lcrrr}
\hline Data & Espécies & Machos & Fêmeas & Total \\
\hline 8 a 9 de maio de 2000 & L intermedia & 36 & 273 & 309 \\
\hline 29 a 30 de junho de 2000 & L intermedia & 1 & 118 & 119 \\
& L. lutziana & - & 2 & 2 \\
& B.sp & - & 1 & 1 \\
\hline 5 a 6 de outubro de 2000 & L intermedia & 883 & 2513 & 3396 \\
& L lutziana & - & 2 & 2 \\
& L sordelli & - & 1 & 1 \\
& B. sp & 1 & 2 & 3 \\
\hline 17 a 18 de janeiro de 2001 & L intermedia & 127 & 903 & 1030 \\
\hline Total & & 1.048 & 3.815 & 4.863
\end{tabular}

partir das 18 horas e 15 minutos até às 4 horas e 15 minutos do dia seguinte. Foram capturados 309 exemplares, sendo todos L intermedia (Tabela 2 e Figura 2). As 4 armadilhas CDC foram instaladas às 16 horas e recolhidas às 7 horas do dia seguinte. Estas capturaram 13 espécimens, todos L intermedia (Tabela 1).

$\mathrm{Na}$ captura realizada das 16 horas do dia 29/06/2000 às 7 horas do dia 30/06/2000, a temperatura local oscilou de $19^{\circ} \mathrm{Ca}$ $11^{\circ} \mathrm{C}$, a umidade relativa do ar ficou entre $60 \%$ e $75 \%$, sem precipitação atmosférica diária e mensal. Nesta captura foram coletados flebotomíneos na armadilha tipo Shannon a partir das 18 horas e 10 minutos até as zero hora e 10 minutos, totalizando 122 espécimens, dos quais, 119 pertenciam a Lintermedia (Tabela 2 e Figura 2). As armadilhas CDC durante as 15 horas que ficaram instaladas capturaram apenas 9 exemplares, todos pertencentes a L intermedia (Tabela 1 ).

Na captura realizada das 16 horas do dia 05/10/2000 às 7 horas do dia 06/10/2000, a temperatura local ficou entre $22^{\circ} \mathrm{C}$ e $19^{\circ} \mathrm{C}$, com umidade relativa do ar entre $70 \%$ a $95 \%$ sem precipitação atmosférica diária, mas, com precipitação mensal de $16,7 \mathrm{~mm}$. Nesta captura obteve-se flebótomos na armadilha de Shannon a partir das 18 horas e 40 minutos às 6 horas e 40 minutos. Foram capturados 3.402 exemplares, sendo 3.396 de L intermedia (Tabela 2 e Figura 2). Nas armadilhas de CDC num período de 15 horas capturaram-se 1.365 espécimens de L intermedia (Tabela 1).

Na última captura, nos dias 17/01/2001 a 18/01/2001, a temperatura local variou de $24^{\circ} \mathrm{C}$ a $21^{\circ} \mathrm{C}$ e a umidade relativa do ar ficou entre 80 e 100\%, sem precipitação atmosférica diária, porém com precipitação mensal de $256,6 \mathrm{~mm}$. Nesta captura capturou-se flebótomos a partir das 20 horas e 20 minutos às 5 horas e 20 minutos ( horário de verão) e foram capturados na armadilha de Shannon 1.030 exemplares, todos de L intermedia (Tabela 2 e Figura 2). Já nas 15 horas que as armadilhas CDC ficaram instaladas, capturaram 290 espécimens de L intermedia (Tabela 1).

\section{DISCUSSÃ0}

Das 8 espécies de flebotomíneos encontradas, 99,9\% foram de L intermedia, isso porque este local se encontra totalmente alterad ${ }^{819}$, com algumas árvores nativas, plantio de fruteiras mas, 0 que pode ter influenciado nesta abundância de espécimens sem dúvida nenhuma, foi a quantidade de bananeiras cultivadas neste local, favorecendo um ambiente úmido e sombrio.

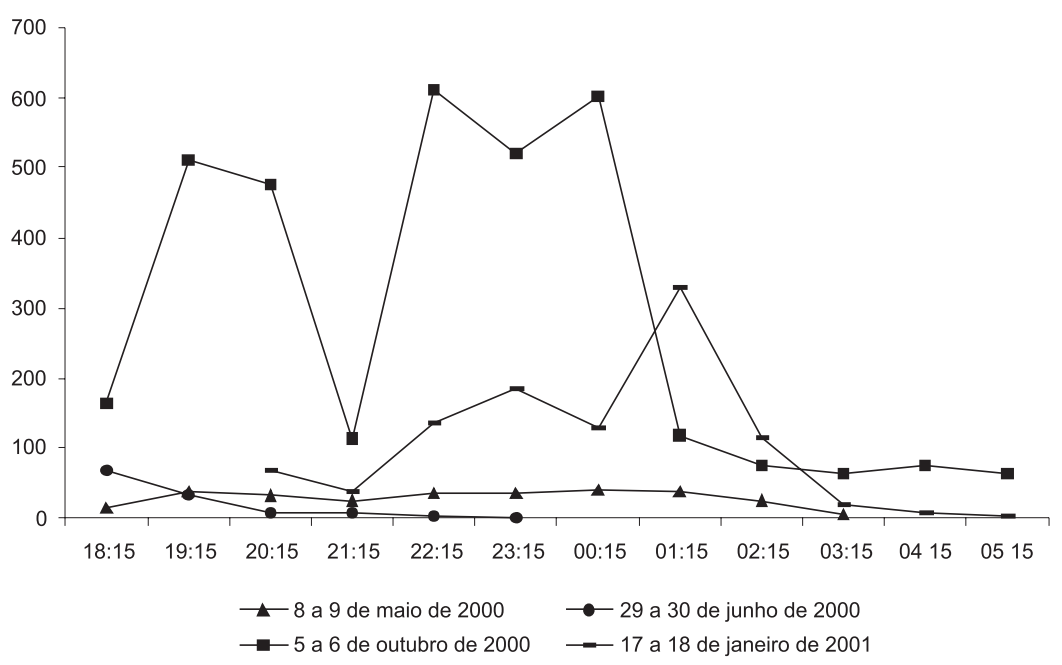

Figura 2 - Números de exemplares de Lutzomyia intermedia capturados em monitoramento de modo contínuo de 15 horas, na armadilha tipo Shannon, em uma área de transmissão da LTA no Município de Uberlândia - MG, 2002. 
Durante 0 período das 4 capturas, observou-se que os flebotomíneos começaram a chegar ao escurecer, mas havendo variações quanto ao horário de desaparecerem conforme demonstrado na Figura 2.

Na primeira captura em 5 de maio de 2000, no primeiro horário os flebotomíneos começaram a aparecer em menor proporção tendo um aumento representativo a partir do segundo horário mantendo-se até às 3 horas e 15 minutos. A partir deste horário houve uma queda acentuada no número de espécimes levando ao desaparecimento total dos insetos no $11^{0}$ horário em diante ( Figura 2). Apesar da temperatura ter oscilado de $21^{\circ} \mathrm{C}$ a $14^{\circ} \mathrm{C}$, observou-se uma grande quantidade de orvalho que caia no local de captura, impedindo que os flebótomos saíssem de seus ecótopos.

Na segunda captura em 29 de junho de 2000, os flebotomíneos tiveram comportamento diferenciado em relação à primeira captura. Neste dia, 0 maior número de espécimens capturados foi no primeiro horário 68 flebotomíneos, diminuindo em 51,5\% (33) no segundo horário, deste para 0 terceiro horário, a diferença foi de 76,5\% (8). Do terceiro horário para 0 quarto praticamente não houve diferença, mas no quarto horário obteve-se apenas 2 flebótomos chegando no quinto horário ( 23 horas 10minutos à zero hora e 10minutos) apenas com um exemplar ( Figura 2).

Este fato pode ter ocorrido devido a temperatura, pois constatou-se que conforme a temperatura foi diminuindo com 0 avanço da noite, também houve diminuição na quantidade de flebótomos capturados. Em Itupeva, região sudeste do Estado de São Paulo, pesquisadores observaram que no mês de junho, houve uma queda brusca no número de flebotomíneos, o que foi relacionada com a possível diminuição da temperatura ${ }^{13}$.

Em 5/10/2000, na terceira captura, capturou-se flebótomos das 18 horas e 40 minutos às 6 horas e 40 minutos. Neste dia, os flebótomos começaram a chegar na armadilha a partir das 18 horas e 40 minutos permanecendo até as 6 horas e 40 minutos. No primeiro horário os insetos chegaram em menor proporção tendo um elevado aumento no segundo e permanecendo no terceiro horário. No quarto horário houve uma queda brusca no número de espécimens, sendo este fato relacionado a ocorrência de ventos moderados que surgiram neste horário, fatores já citados como interferentes na atividade de flebotomíneos ${ }^{1}$. No quinto, sexto e sétimo horários, os espécimens voltaram a aparecer em grande quantidade novamente. Do sétimo horário para 0 oitavo, observou-se uma queda de $80,4 \%$ do número de espécimens. Do oitavo para o nono horário, a diferença ficou em $36,4 \%$. A partir do nono horário, a quantidade de flebótomos manteve-se praticamente a mesma até 0 último horário (Figura 2). Outros pesquisadores ${ }^{3} 19$ também capturaram L intermedia das 18 horas às 6 horas.

Este grande número de flebotomíneos capturados pode estar relacionado às condições ambientais propícias deste dia ( temperatura e umidade do ar elevadas com ventos moderados apenas em um dos horários e sem orvalho) e provavelmente a sua reprodução ( grande quantidade de flebótomos na cópula) .
Aúltima captura, em 17/01/2001, ocorreu em época que favoreceria uma grande quantidade de flebótomos, mas a alta pluviosidade deste período pode ter impedido os flebotomíneos de saírem de seus ecótopos, fator já reconhecido como interferente na atividade dos flebotomíneos ${ }^{4}$.

A L intermedia predominou nas 4 capturas, com 99,7\% do total, durante 0 período de pesquisa; porém, com uma maior $(75,5 \%)$ quantidade da espécie no mês de outubro que precede 0 período chuvoso, levando-nos a considerar que neste período pode ocorrer um maior risco de infecção pela doença em nosso Município. No mês frio e seco, como junho, o perigo de transmissão da doença ficaria restrito antes da zero hora, o que não estaria ocorrendo nos demais meses, quando se obteve flebótomos em todos os horários.

\section{REFERÊNCIAS BIBLIOGRÁFICAS}

1. Aguiar GM, Soucasaux T. Aspectos da ecologia dos flebótomos do Parque Nacional da Serra dos Órgãos, Rio de Janeiro. IV - Freqüência mensal em armadilhas luminosas (Diptera, Psychodidae, Phlebotominae). Memórias do Instituto Oswaldo Cruz 80: 465-482, 1985.

2. Baccaro CAD. Estudos geomorfológicos do município de Uberlândia. Sociedade \& Natureza 1: 17-21, 1989.

3. Condino MLF, Sampaio SMP, Henriques LF, Galati EAB, Wanderley DMV, Corrêa FMA. Leishmaniose tegumentar americana: flebotomíneos de área de transmissão no município de Teodoro Sampaio, região sudoeste do Estado de São Paulo, Brasil. Revista da Sociedade Brasileira de Medicina Tropical 31: 355-360, 1998.

4. Domingos MF, Correri-Bruno GC, Ciaravolo RMC, Galati EAB, Wanderley DMV, Corrêa FMA. Leishmaniose tegumentar americana: flebotomíneos de área de transmissão, no município de Toledo, região sul do Estado de São Paulo, Brasil. Revista da Sociedade Brasileira de Medicina Tropical 31: 425-432, 1998.

5. Fundação Nacional de Saúde. Controle, diagnóstico e tratamento da leishmaniose visceral ( calazar). Brasília, 1996.

6. Fundação Nacional de Saúde. Centro Nacional de Epidemiologia. Guia de vigilância epidemiológica. Brasília, 1998.

7. Fundação Nacional de Saúde. Manual de controle da leishmaniose tegumentar americana. Brasília, 2000.

8. Galati EAB, Nunes VLB, Dorval MEC, Oshiro ET, Cristaldo G, Espíndola MA, Rocha HC da, Garcia WB. Estudos dos flebotomíneos (Diptera, Psychodidae), em área de leishmaniose tegumentar, no Estado de Mato Grosso do Sul, Brasil. Revista Saúde Pública 30: 115-128, 1996.

9. Gomes AC, Galati EAB. Aspectos ecológicos da leishmaniose tegumentar americana: 7. Capacidade vetorial flebotomínea em ambiente florestal primário do Sistema da Serra do Mar, região do Vale do Ribeira, Estado de São Paulo, Brasil. Revista Saúde Pública 23: 136-142, 1989.

10. Lemos JC, Lima SC, Pajuaba Neto AA,Casagrande B, Vieira GSS, Ferrete JÁ, Magalhães MJ0. Encontro de Lutzomyia longipalpis na área de implantação da Usina Hidrelétrica Capim Branco I, na bacia do rio Araguari, no município de Uberlândia, Minas Gerais - Brasil. Disponível em: <www.ig.ufu.br/revista/volume11/ artigo12_vol11.pdf>. Caminhos de Geografia 11:186-198, 2004.

11. Lima SC, Rosa R, Feltran Filho A. Mapeamento do uso do solo no município de Uberlândia-MG, através de imagens TM/LANDSAT. Sociedade \& Natureza 1: $127-145,1989$.

12. Luz ZMP, Pimenta DN, Cabral ALLV, Fiúza VOP, Rabelo A. A urbanização das leishmanioses e a baixa resulutividade diagnóstica em municípios da Região Metropolitana de Belo Horizonte. Revista da Sociedade Brasileira de Medicina Tropical 34: 339-345, 2001. 
13. Mayo RC, Casanova C, Macarini LM, Pignatti MG,Rangel 0, Galati EAB, Wanderley DMV, Corrêa FMA. Flebotomíneo (Diptera, Psychodidae) de área de transmissão de leishmaniose tegumentar americana, no município de Itupeva, região sudeste do Estado de São Paulo, Brasil. Revista da Sociedade Brasileira de Medicina Tropical 31: 339-345, 1998.

14. Rosa R, Lima SC, Assunção WL. Abordagem preliminar das condiç̃oes climáticas de Uberlândia (MG). Sociedade \& Natureza 3: 91-108, 1989.

15. Schiavini I, Araújo GM. Considerações sobre a vegetação da reserva ecológica do Panga (Uberlândia). Sociedade \& Natureza 1: 61-65, 1989.

16. Serviços de Informação. Guia Sei: serviços-endereços-informações 2002. SABE, Uberlândia, 2002.
17. Shannon RC. Methods for collecting and feeding mosquitoes in jungle yellow fever studies. American Journal of Tropical Medicine 19:131-138, 1939.

18. Sudia WD,Chamberlain RW. Battery operated light trap, an improved model. Mosquito News 22: 126-129, 1962.

19. Teodoro U, La Salvia Filho V, Lima EM, Spinosa RP, Barbosa OC, Ferreira MEMC, Silveira TGV. Flebotomíneos em área de transmissão de leishmaniose tegumentar na região norte do Estado do Paraná - Brasil variação sazonal e atividade noturna. Revista Saúde Pública 27: 190-194, 1993. 